\title{
Complications and short-to-midterm results in a case series of 52 CUE procedures using a modified caudo-medial approach
}

\author{
Karol Bayer ${ }^{1 *}$, Philipp Winkels ${ }^{2}$, Angelo Alessandro Andreoni ${ }^{3}$, Philipp Schmierer ${ }^{4}$, Thomas Rohwedder ${ }^{1}$, \\ Antonio Pozzi ${ }^{4}$ and Peter Böttcher ${ }^{1}$ \\ ${ }^{1}$ Small Animal Clinic, Free University of Berlin, Berlin, Germany \\ ${ }^{2}$ Vetacare, Erftstadt, Germany \\ ${ }^{3}$ Ennetsee Small Animal Clinic, Hünenberg, Switzerland \\ ${ }^{4}$ Clinic for Small Animal Surgery, Vetsuisse Faculty University of Zurich, Zurich, Switzerland
}

\begin{abstract}
Background: Medial coronoid process disease is the most common manifestation of canine developmental elbow disease which can progress to a more severe medial compartment disease (MCompD) characterized by fullthickness cartilage loss of the medial coronoid process and the medial humeral condyle. Among others, the "Canine Unicompartmental Elbow" (CUE) has been reported to be an effective treatment strategy for MCompD, with full in $47.6 \%$ and acceptable function in $43.7 \%$ at 6 months or later of follow-up.

Aim: To report on our clinical experiences with the CUE system using the caudo-medial approach in terms of both complications and functional outcome.

Methods: Medical records of dogs that underwent CUE procedure using a caudo-medial approach over a 3-year period were retrospectively reviewed. This covered epidemiological data, bi-planar radiographs, subjective gait analysis, owner questionnaire, surgical reports, as well as second-look arthroscopic findings when available.

Results: In total, 52 CUE procedures were performed in 44 dogs with a median age of 8.0 years (IQ: 5.0-10.0) and a median bodyweight of $31.9 \mathrm{~kg}$ (ranging 20-48 kg) at the surgery. Four cases never return for follow-up, but were included in the analysis to increase the number of cases with pre- and intra-operative data. Mean follow-up time available for the remaining 48 cases was 7.1 (SD: 5.2) months. Radiographic derived implant positioning and alignment proved to be satisfactory in the sagittal plane but parallelism in the frontal plane was only present in three cases. Second-look arthroscopy in five cases with delayed or disappointing functional improvement showed evidence of implant-related contact lesions and progressive erosion of the medial coronoid area in three elbows. Overall, complications occurred in 11 cases (21\%), being major in eight (15\%) and minor in three (6\%). Major complications included refractory pain and lameness 6 to 12 months postoperatively in five cases. At last follow-up, 12 cases (25\%) were considered to have full function, 35 cases (73\%) acceptable function, and in one case, the function was considered unacceptable. As the only variable related to functional outcome, age had a negative predictive value for full function.

Conclusion: The CUE procedure appears to be an effective treatment option for patients with MCompD. Older dogs might be at risk of having an inferior clinical outcome when compared to young patients. The reason for this is unknown and will have to be evaluated in future studies. Compared to a CUE case series of 103 elbows operated through a medial approach, using a caudo-medial approach decreased the incidence of approach-related complication. Nevertheless, the functional outcome in the current case series was less favorable than previously reported. These conflicting findings as well as the occurrence of potentially implant mechanical conflict at the medial joint compartment despite CUE warrants further studies.
\end{abstract}

Keywords: Canine unicompartmental elbow, CUE, Medial compartment disease.

\section{Introduction}

Medial coronoid process disease is the most common manifestation of canine developmental elbow disease and a frequent cause of thoracic limb lameness (Van Ryssen and van Bree, 1997; Fitzpatrick et al., 2009a; Smith et al., 2013). This pathology can progress to a more severe medial compartment disease (MCompD) (Van Ryssen and van Bree, 1997; Vermote et al., 2009; Franklin et al., 2014), which is characterized by full-thickness cartilage loss of the medial coronoid process (MCP) and medial humeral condyle (modified Outerbridge scores 4 and 5) (Fitzpatrick et al., 2009b; Farrell et al., 2014; Franklin et al., 2014; Coppieters et al., 2015).

Among others, the "Canine Unicompartmental Elbow" (CUE) Arthroplasty System ${ }^{\circledR}$ (Arthrex Vet Systems, Naples, FL) has been reported to be an effective treatment strategy for MCompD, with full in $47.6 \%$ and acceptable function in $43.7 \%$ at 6 months or later of follow-up (Franklin et al., 2014; Cook et al., 2015). The 
CUE is a unicompartmental joint replacement system, which utilizes press-fit humeral and ulnar implants to partially resurface the eburnated joint surfaces of the medial joint compartment, with the goal to eliminate bone on bone contact throughout and slightly beyond the normal stance phase. Because of the slightly proud implantation of the humeral implant (HImpl), the CUE also addresses the collapse of the medial jot compartment associated with MCompD. Because the clinical success of CUE is based on the proper implant on implant contact during weight-bearing, any malpositioning or mal-orientation of one or both implants may lead to the unsatisfactory clinical outcome or even major complications. These include implant loosening and implant wear or grinding of the HImpl into the articulating ulnar joint surface. The latter may occur especially due to insufficient implant overlap or oblique orientation of the HImpl with respect to the ulnar joint surface. Despite these potential concerns, so far CUE reported complications are predominately associated with the surgical approach, which was either via osteotomy of the medial epicondyle or tenotomy and desmotomy (Cook et al., 2015). This is why several CUE surgeons have adopted a caudo-medial approach to the elbow joint (Amman and Wendelburg, 2010; Smith et al., 2013) with the expectation of lower complication rates and reduced postoperative morbidity (Walmsley and Fitzpatrick, 2018; Winkels and Busch, 2018).

The aim of this study is to report on our clinical experiences with the CUE system using the caudomedial approach in terms of both complications and functional outcome. Because we hypothesize that position and alignment of the implant could influence the functional outcome as well as rate and type of complications, we report on the radiographic assessment of implant position and alignment.

\section{Material and Methods \\ Surgical technique using a modified caudo-medial approach}

The dog is positioned in dorsal recumbency with a pad underneath the elbow as previously described (Franklin et al., 2014). The caudal ulnar spine, medial humeral epicondyle, and medial olecranon ridge are palpated (Fig. 1A). The skin incision starts slightly medial to the spine at about $30 \%$ of the proximal spine length. The incision continues proximally between the olecranon and medial epicondyle to the point over the olecranon ridge along the medial border of the triceps muscle. The antebrachial fascia is exposed and incised directly over the ulnar spine (Fig. 1B). The incision continues proximally towards the olecranon. The flexor carpi ulnaris muscle is lifted subperiosteally up to its attachment to the olecranon ridge (Fig. 1C). Its origin is either released via tenotomy (Fig. 1D) or an osteotomy of the olecranon ridge (Fig. 1E). The ulnar head of the flexor carpi ulnaris muscle can then be retracted cranially. This allows exposure of the caudal aspect of the elbow joint and caudo-medial joint capsule, which can be incised with a stab incision. The arthrotomy is extended proximally deep into the olecranon fossa with transection of the olecranon ligament as well as distally, up to the level of the medial collateral ligament (Fig. 1F). A blunt Hohmann retractor, inserted just medial to the medial collateral ligament in the front of the radial head, retracts all soft tissues cranially (Fig. 1G), including the ulnar nerve. Biceps ulnar release procedure (BURP) along the caudal aspect of the medial collateral ligament improves the opening of the joint space when exerting supination of the ulna (Fig. 1H). A medium-sized bone clamp at the level of the olecranon acts as a lever arm for further pronation of the ulna, improving access to the medial joint compartment (Fig. 1I). Once proper access to the joint is achieved, implantation of the HImpl and UImpl is identical to what has been previously described (Franklin et al., 2014).

After positioning of the implants, the osteotomy is reduced and fixed with cortical screws or K-wires. In case of tenotomy, the flexor carpi ulnaris muscle is re-apposed and fixed with a monofilament absorbable suture either by anchoring in the remaining periosteum or through transosseous tunnels. The incision is then closed routinely in three layers. Suturing of the joint capsule is not performed.

\section{Inclusion criteria}

Medical records of dogs that underwent CUE procedure using the caudo-medial approach between February 2016 and June 2018, from four different institutions were reviewed. The indication to perform the CUE procedure had been advanced to end-stage $\mathrm{MCompD}$, being defined as modified Outerbridge Score 4 to 5 at the trochlea and MCP. Only ECVS Diplomates with advanced CUE experiences were involved in these procedures.

Only cases with complete medical records, including preoperative and postoperative medio-lateral (ML) and cranio-caudal (CrCd) radiographic views and arthroscopic evaluation prior to CUE implantation, were considered. In the case of bilateral CUE, each elbow was considered a separate procedure.

\section{Data collection}

Epidemiological data (breed, sex, body weight, and age at the time of surgery), a variation of caudo-medial approach performed (osteotomy vs. tenotomy), size, and number of implants used were recorded.

Absence of gross cartilage pathology at the lateral joint compartment was mandatory for CUE implantation. However, cartilage loss on the medial oblique aspect of the radial head was considered to be part of the MCompD and did not result in the exclusion of the case. Degree of degenerative joint disease (DJD) was scored preoperatively and during follow-up according to the International Elbow Working Group Score (IEWG Score) (Ohlerth et al., 2016).

Ethical approval

All surgeries and data collection were carried out with the patient's owner agreement. 


\section{Implant position}

Immediate postoperative views were used for measurements of the implant position and their overlap. All measurements and evaluation were made using OsiriX Lite (Pixmeo, Bernex, Switzerland) and ImageJ (public domain, www.imagej.nih.gov/ij).
Parallelism of the implants contact surface in the coronal plane was measured on the $\mathrm{CrCd}$ view. First, a line parallel to the surface of the HImpl and a second line identical with the longitudinal axis of the elliptically projected metallic base of the UImpl were drawn. The angle between those two lines is reported as

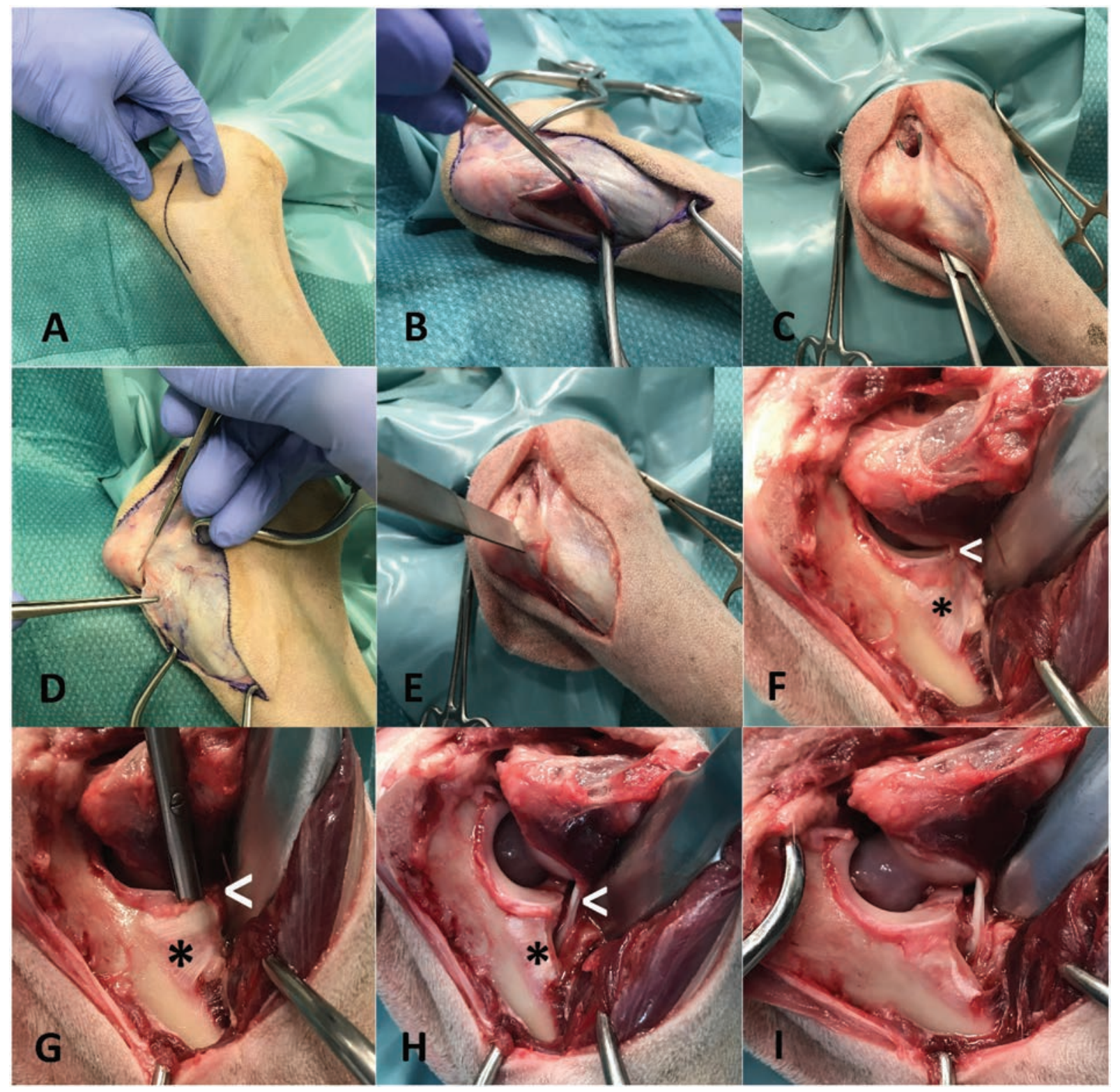

Fig. 1. Caudo-medial approach to the elbow joint: (A) skin incision between medial humeral epicondyle and olecranon ridge extending about 10 distally along the ulnar spine; (B) incision of the antebrachial fascia directly onto the ulnar spine; (C) humeral head of flexor carpi ulnaris muscle is lifted subperiosteally up to its attachment at the olecranon ridge, tunneling with a Metzenbaum scissor, dissecting the antebrachial facia along the medial border of the triceps muscle; (D) tenotomy of the flexor carpi ulnaris muscle; (E) osteotomy of the olecranon ridge with attached flexor carpi ulnaris muscle; (F) cranial retraction of flexor carpi ulnaris muscle with broad Hohmann retractor resting cranial on the radius, allowing visualization of medial collateral ligament and biceps ulnar tendon; $(\mathrm{G})$ tunneling of the biceps ulnar tendon caudal to the medial collateral ligament for isolation of the biceps tendon and subsequent release; $(\mathrm{H})$ biceps ulnar release caudal to the medial collateral ligament, olecranon ligament has also been transected (not shown); and (I) application of curved medium sized bone clamp at the level of the olecranon, acting as a lever for pronation of the ulna. white arrow —medial collateral ligament; asterix — biceps ulnar tendon. 
medial or lateral opening angle, depending on whether the angle was open towards the medial or lateral aspect of the joint (Fig. 2).

Medio-lateral position of the HImpl in the medial humeral trochlea was also determined on the $\mathrm{CrCd}$ views. Implant position was expressed as a percentage of the distance between the trochlear notch to the center of the HImpl and the total width of the medial humeral trochlea.

Cranio-caudal overlap of both implants was measured on ML views (Figs. 3 and 4).

First, the humeral longitudinal axis was drawn, as a line connecting the midpoints between both humeral cortices at $50 \%$ and distal $30 \%$ of its length. The radial longitudinal axis was determined by applying the same principle. A goniometer template was then positioned with each arm parallel to the respective longitudinal axis with the center of rotation at the center of the humeral condyle. Cranial and caudal edges of the implants were then marked on the template (Fig. 3). While the humeral arm of the template remained fixed, the radial arm with marked UImpl edges was first brought into full extension, positioning the ulnar implant caudal

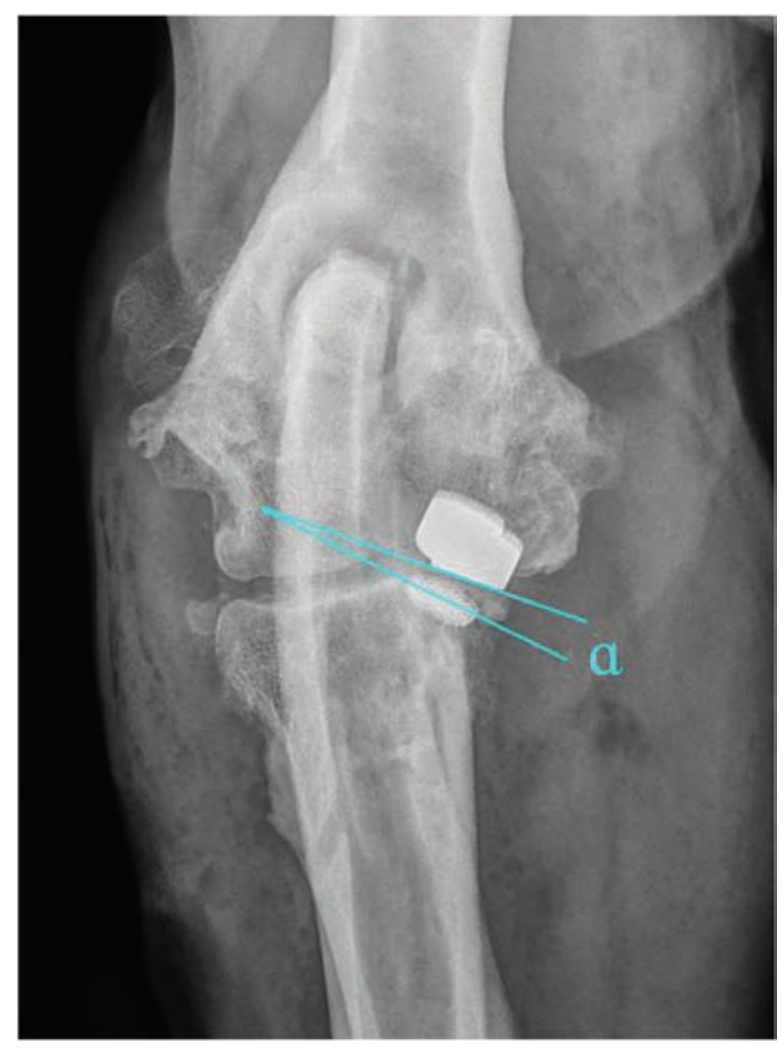

Fig. 2. Determination of implant parallelism showing a medial opening angle $\alpha=7^{\circ}$. First-line lies parallel to the surface of the HImpl and a second line is identical with the longitudinal axis of the elliptically projected metallic base of the UImpl. to the humeral one and then gradually flexed back to full flexion in order to mimic the full range of elbow motion. Extension angles for six elbow positions were recorded (Fig. 4):

1. Extension angle with no overlap between UImpl and HImpl, with the HImpl just cranial to the ulnar implant (Fig. 4A).

2. Extension angle with 50\% UImpl overlap (Fig. 4B).

3. Extension angle with the caudal part of the HImpl overlapping $100 \%$ of the UImpl (Fig. 4C).

4. Extension angle with the cranial part of the HImpl overlapping the whole UImpl (Fig. 4D).

5. Extension angle with 50\% UImpl coverage (Fig. 4E)

6. Extension angle with no overlap between UImpl and HImpl, with the HImpl just caudal to the ulnar implant (Fig. 4F).

The difference between the largest and the smallest extension angle measured represents the range of elbow motion with implant overlap and is termed the implant

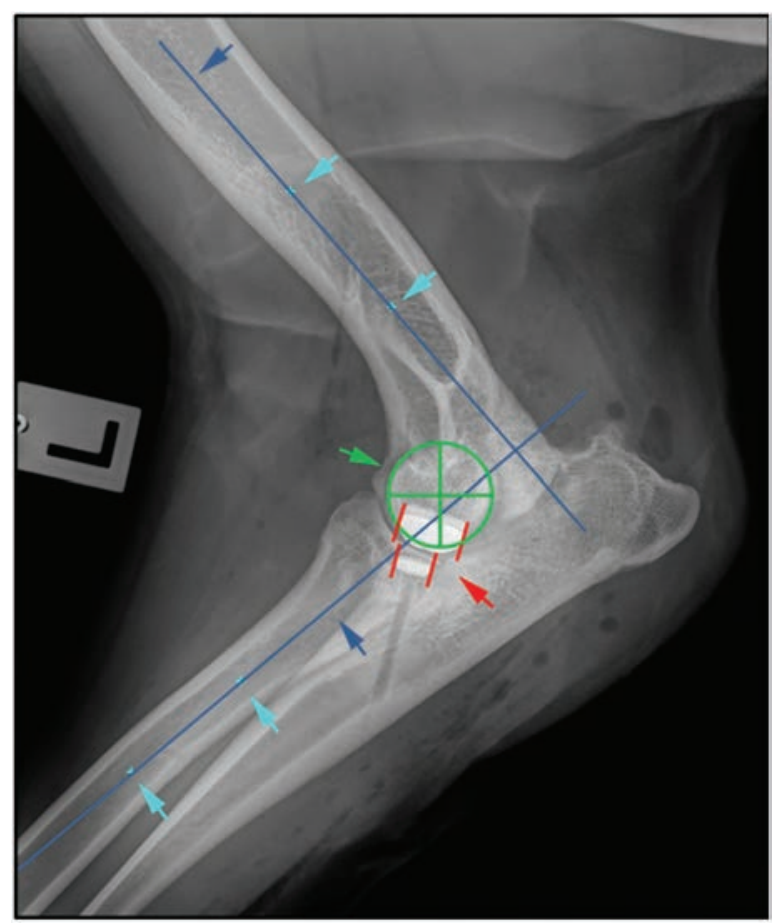

Fig. 3. Determination of long bone axis as well as joint rotation center for measurement of cranio-caudal humeroulnar implant overlap turquoise arrows: midpoints between cortices at $50 \%$ and $30 \%$ of the humeral and radial bone length; blue arrows: longitudinal bone axis of humerus and radius; green cross: center of elbow joint rotation, identical with center of humeral condyle; red lines: cranial and caudal edge of the humeral and ulnar implants. 


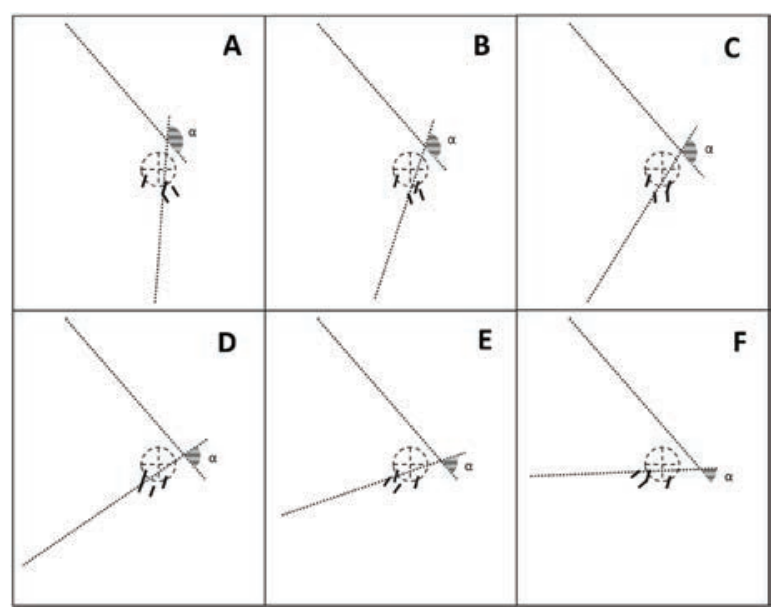

Fig. 4. Measurement of simulated elbow joint extension angle for six states of cranio-caudal humero-ulnar implant overlap, starting with full extension. (A) No overlap between ulnar and humeral implant, with the ulnar implant just caudal to the humeral one; (B) coverage of the cranial half of the ulnar implant (50\%); (C) largest extension angle with 100\% ulnar coverage; (D) smallest extension angle with $100 \%$ ulnar coverage; (E) coverage of the caudal half of ulnar implant (50\%); and (F) no overlap between ulnar and humeral implant, with the ulnar implant just cranial to the humeral one. $\alpha$ : measured extension angle.

contact phase span (ICPS). In cases with multiple ulnar implants, these measurements were repeated, with the caudal border of the most caudal UImpl and the cranial border of the most cranial UImpl set as reference.

\section{Follow-up}

Follow-up examinations were performed 2, 6, and 12 months after surgery and then annually. Clinical variables recorded at each follow-up examination included the presence of signs of pain or discomfort on the manipulation of the elbow, range of motion (ROM), and lameness grade. Clinical outcome was defined asfull function, acceptable function, and unacceptable function according to Cook et al. (2010).

A minimum of two orthogonal radiographs were obtained at each follow-up examination. Radiographs were scored according to the IEWG to monitor the progression of DJD. Implant associated complications such as loosening, as well as any other radiographic abnormalities were also recorded. Implant loosening was defined by the presence of radiolucency around the implant in $\geq 60 \%$ of the bone-implant interface, following the guidelines for cementless hip prostheses cups (Hanson et al., 2006).

At the time of summarization of the study results, the LOAD questionnaire was sent to owners to assess the actual clinical status of their dog. Clinical signs were defined as "mild" for LOAD scores from 0 to 10, "moderate" from 11 to 20, "severe" from 21 to 30 , and "extreme” for scores from 31 to 52 (Walton et al., 2017).

\section{Complications}

Any complications were documented and defined with respect to the time of onset. According to proposed outcome criteria by Cook et al. (2010), these onsets were defined as intraoperative, postoperative ( $0-3$ months), short-, mid-, and long-term. Severity of complications was defined as minor, major, or catastrophic. In extension to the proposed nomenclature, we also defined refractory pain and lameness 6 to 12 months after CUE implantation to be a major complication.

Statistical analysis

Continuous data were tested for normality using D'Agostino-Pearson test and expressed as mean and standard deviation or median and 75\% interquartile range in case of non-normality. Progression of radiographically scored osteoarthritis (IEWG score) was analyzed using the Wilcoxon test for paired samples. Difference in LOAD scale depending on functional outcome was tested using the independent $t$-test. Association of age, body weight, pre-operative IEWG score, modification of caudo-medial approach, time of follow-up, size, and positioning/orientation of the implants with the functional outcome as well as the occurrence of any form of complication were tested using logistic regression analysis. For all tests, $\alpha$ was set to 0.05 .

\section{Results}

Inclusion criteria were met for 52 CUE procedures in 44 dogs, out of which 26 were male (7 castrated, 19 intact) and 18 female (13 spayed, 5 intact). Median age at surgery was 8.0 years (IQ: 5.0-10.0) and median body weight was $31.9 \mathrm{~kg}$ (ranging 20-48 kg). In total, 16 breeds were represented, with Labradors and Retrievers accounting for $48 \%$.

In 36 elbows, a tenotomy of the flexor carpi ulnaris muscle was performed as part of the caudo-medial approach with osteotomy of the olecranon ridge being performed in the remaining 16 cases.

\section{Implants and position}

Sizes and number of implants used are summarized in Figure 5. The use of one large humeral and one large ulnar implant was the common combination $(n=30)$. In five elbows, more than one UImpl was implanted. The median deviation from true humero-ulnar implant parallelism was $10.8^{\circ}$ (IQ: $5.5^{\circ}-17.0^{\circ}$ ). In only three cases were the desired parallelism of $\pm 1^{\circ}$ achieved. In 41 cases, a median medial opening angle of $14.0^{\circ}$ (IQ: $\left.8.3^{\circ}-18.0^{\circ}\right)$ was found with the remaining cases having a median lateral opening angle of $3.0^{\circ}$ (IQ: $1.8^{\circ}-4.6^{\circ}$ ). The mean medio-lateral position of the HImpl was centered along the width of the humeral trochlear (50.9\%; SD: $10.1 \%$ ).

The results of cranio-caudal implant overlap measurements in the sagittal plane are illustrated in Table 1.

ROM values for $50 \%$ and $100 \%$ overlap for one, two, or three implants, respectively, are illustrated in Table 2. 


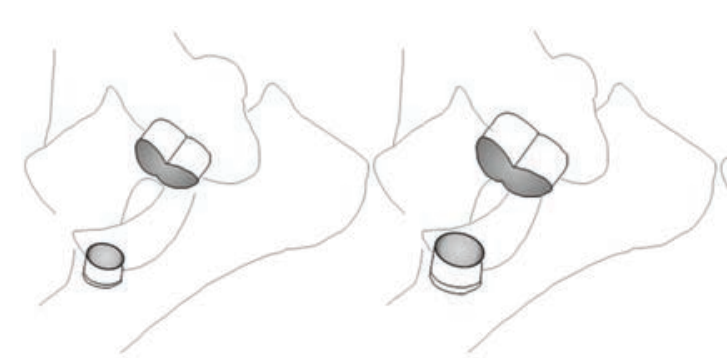

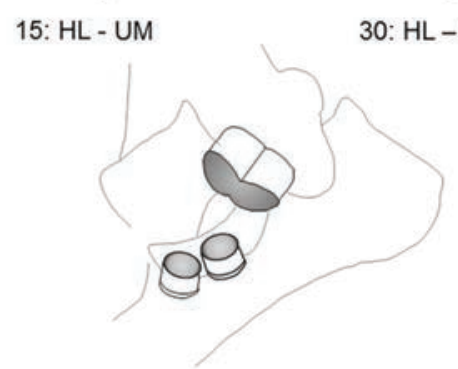

3: $\mathrm{HL}-2 \times \mathrm{UM}$

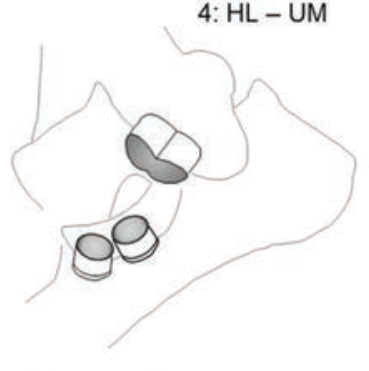

1: $H M-2 x U M$

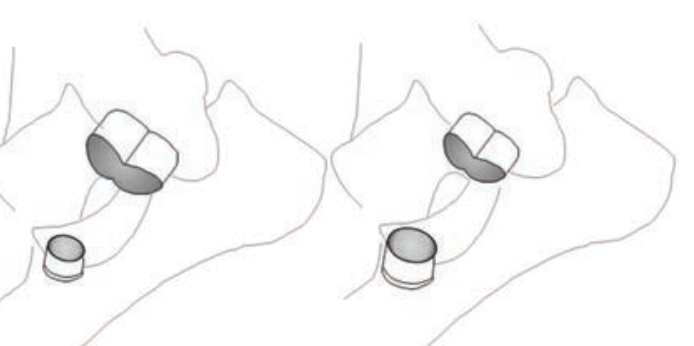

3: HM - UL

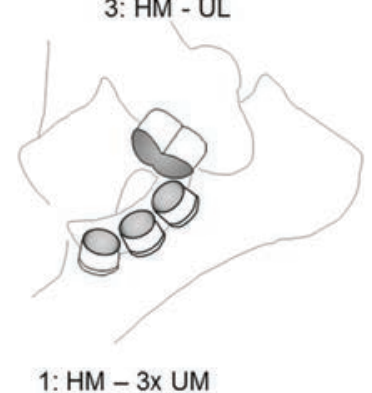

Fig. 5. Schematic drawing of sizes and combinations of implants. The combination of one large humeral and one large ulnar implant was the most common implant set used.

Table 1. Cranio-caudal Implant Overlap. Starting with full extension, the overlap of the implants in the sagittal plane began at $169.1^{\circ}$ (SD: $3.5^{\circ}$ ) of elbow extension. Fifty percent of the UImpl were covered by HImpl at $153.8^{\circ}$ (SD: $24.0^{\circ}$ ) of extension. Full coverage of the UImpl was present between $136.3^{\circ}$ (SD: $22.6^{\circ}$ ) and $103.7^{\circ}$ (SD: $20.4^{\circ}$ ). Implant overlap was reduced again to $50 \%$ at an extension angle of $88.9^{\circ}$ (SD: $19.5^{\circ}$ ) and coverage was lost at an angle of $72.6^{\circ}$ (SD: $20.3^{\circ}$ ).

\begin{tabular}{cccccc}
\hline Implant overlap & $\mathbf{0 \%}$ & $\mathbf{5 0 \%}$ & $\mathbf{1 0 0 \%}$ & $\mathbf{5 0 \%}$ & $\mathbf{0 \%}$ \\
\hline Extension angle & $169.1^{\circ}\left(\mathrm{SD}: 3.5^{\circ}\right)$ & $153.8^{\circ}\left(\mathrm{SD}: 24.0^{\circ}\right)$ & $\begin{array}{c}136.3^{\circ}\left(\mathrm{SD}: 22.6^{\circ}\right) \\
103.7^{\circ}\left(\mathrm{SD}: 20.4^{\circ}\right)\end{array}$ & $88.9^{\circ}\left(\mathrm{SD}: 19.5^{\circ}\right)$ & $72.6^{\circ}\left(\mathrm{SD}_{2} 20.3^{\circ}\right)$ \\
\hline
\end{tabular}

Table 2. Implant overlap ROM. With one UImpl overlap of 50\% or $100 \%$ was achieved within a ROM of $58.5^{\circ}$ (IQ: $54.0^{\circ}-66.0^{\circ}$ ) and $31.0^{\circ}$ (IQ: $26.0^{\circ}-35.0^{\circ}$ ), respectively. With two UImpl this increased to $96.5^{\circ}$ (IQ: $82.0^{\circ}-111.0^{\circ}$ ) and $40.8^{\circ}$ (IQ: $26.0^{\circ}-55.5^{\circ}$ ), respectively, while three UImpl resulted in a ROM with $50 \%$ and $100 \%$ of overlap at $106.0^{\circ}$ (IQ: $89.0^{\circ}-123.0^{\circ}$ ) and $55.0^{\circ}$ (IQ: $42.0^{\circ}-68.0^{\circ}$ ), respectively.

\begin{tabular}{ccc}
\hline ROM & ROM for $\mathbf{5 0 \%}$ Overlap & ROM for 100\% Overlap \\
\hline 1 UImpl & $58.5^{\circ}\left(\mathrm{IQ}: 54.0^{\circ}-66.0^{\circ}\right)$ & $31.0^{\circ}$ (IQ: $\left.26.0^{\circ}-35.0^{\circ}\right)$ \\
\hline 2 UImpl & $96.5^{\circ}\left(\mathrm{IQ}: 82.0^{\circ}-111.0^{\circ}\right)$ & $40.8^{\circ}$ (IQ: $\left.26.0^{\circ}-55.5^{\circ}\right)$ \\
\hline 3 UImpl & $106.0^{\circ}\left(\mathrm{IQ}: 89.0^{\circ}-123.0^{\circ}\right)$ & $55.0^{\circ}\left(\mathrm{IQ}: 42.0^{\circ}-68.0^{\circ}\right)$ \\
\hline
\end{tabular}

\section{Radiographic evaluation}

The distribution of IEWG scores over time is illustrated in Figure 6. There was no significant change in IEWG score from pre-operative to the last follow-up. No evidence of implant loosening or subsidence was noted on any follow-up radiograph.

\section{Clinical outcome}

The last clinical follow-up in 48 cases was performed 7.1 (mean, SD: 5.2) months post-operatively. Four out of 52 cases never returned to any form of re-check. In 16 cases, follow-up was shorter than the recommended $\geq 6$ months period. Out of the 48 cases with follow-up, 12 cases (25\%) were considered to have full function, 35 cases (73\%) acceptable function, and in one case, the function was considered unacceptable.
LOAD score reported by the owners was available for 19 cases on average 23.7 (SD: 7.6) months postoperatively. With a median LOAD score of 20.1 (SD: 7.0 ), these cases are classified to have "moderate" clinical signs (Walton et al., 2017). In respect to the differentiation into cases with full versus an acceptable function, there was no significant difference in reported LOAD scores.

Except for age $(p=0.05)$, none of the analyzed variables (body weight, pre-operative IEWG score, selected approach-osteotomy vs. tenotomy, size of humeral and ulnar implants, ICPS with full coverage of the UImpl, implant parallelism, time between surgery to last follow-up, and occurrence of any complications) were significantly correlated with functional outcome 


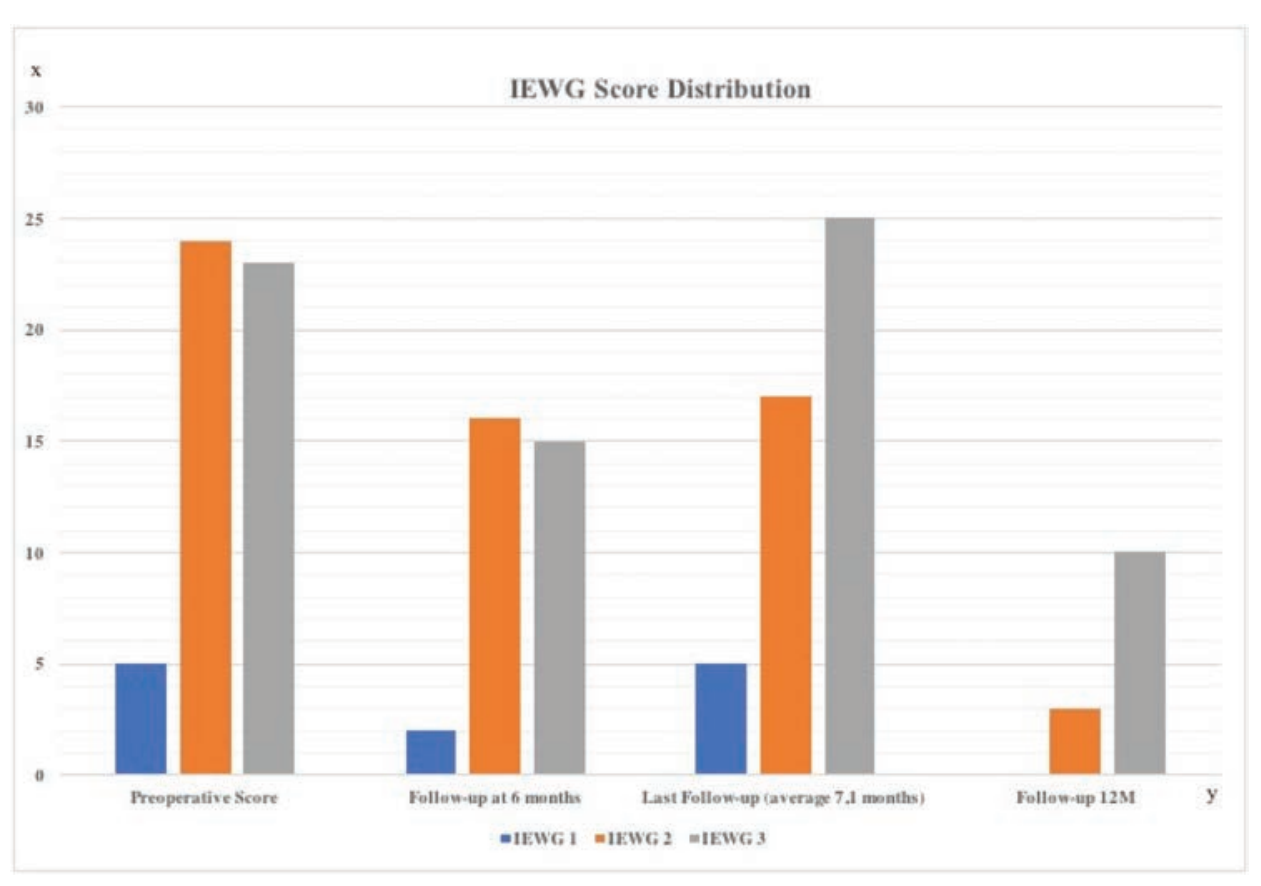

Fig. 6. Distribution of IEWG score. $X$-axis is showing the number of cases and the $Y$-axis is the timeline. The last radiographic control $(n=47)$ was at 7.1 months (SD: 5.2$)$ post-operatively.

in respect to full or acceptable outcome. With increased age, the odds ratio reaches a full functional outcome of 0.59 .

\section{Complications}

Complications occurred in total of 11 cases (21\%), including major complications in eight cases (15\%) and minor complications in three cases (6\%). Major complications occurred intraoperatively in two cases (4\%), one complication occurred perioperatively (2\%), and five cases $(9.5 \%)$ had a mid-term complication. None of the analyzed variables (age at surgery, body weight, pre-operative IEWG score, selected approachosteotomy vs. tenotomy, size implants, cranio-caudal implant coverage (ICPS), and time between surgery to last follow-up) were related to the occurrence of any form of complication.

The most common minor complication was the development of postoperative seroma and/or hematoma around the incision area $(n=2)$; k-wire loosening at the osteotomy site occurred in one case 8 weeks after surgery, which healed uneventfully.

The most common major complication was persistent or recurrent lameness and pain between 6 and 12 months post-operatively $(n=5)$, followed by intra-operative fracture of the radius or ulna (one case each) and septic arthritis together with ulnar implant loosening $(n=1)$. Implant loosening was not evident on the preoperative radiographs.

In all five cases with persistent lameness and pain, a second look arthroscopy was carried out. In two cases, an extension of a full-thickness chondral defect caudal to the ulnar implant was apparent and treated by

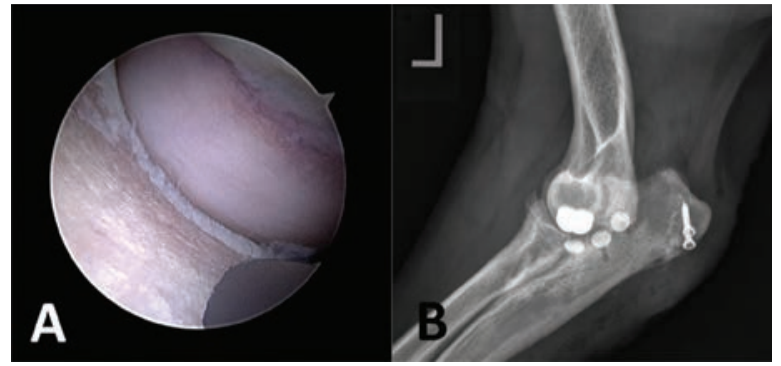

Fig. 7. Case with persistent lameness and pain 6 months after CUE procedure. (A) Second-look arthroscopy showing an extension of full-thickness chondral defect caudal to the ulnar implant and (B) Medio-lateral radiographic view after revision surgery and implantation of two additional mediumsized ulnar implants.

implantation of one $(n=1)$ or two $(n=2)$ additional UImpl just caudal to the initial implant along the ulnar trochlea, using the modified caudo-medial approach and standard CUE implantation technique (Fig. 7). Further healing was uneventful, and at the last followup examination 2 and 10 months after revision surgery, both cases achieved an acceptable outcome with a LOAD score of 18.

In two cases, elbow arthroscopy revealed no significant finding and the source of lameness was never found. In both cases, fibrous cartilage had grown around the humeral and ulnar implants covering the formerly eburnated cartilage areas. In one case, two doses of $2 \mathrm{ml}$ of autologous conditioned plasma (ACP, Arthrex Inc, Naples) were applied intra-articular under sedation to 
the affected elbow 10 days apart. Nine months later, the lameness remained unchanged and the elbow joint was injected with $0.5 \mathrm{ml}$ of Triamcinolone (Triam Injekt, Winthrop Arzneimittel GmbH, Frankfurt am Main, Germany) and injection repeated 2 months later with slight improvement. The second case was treated with an extended activity restriction and short leash walks for another 8 weeks. At the last follow-up examination for these two cases (17 and 8 months after surgery, respectively), lameness had improved compared to the pre-operative examination, but both dogs were still obviously lame at the trot. With the long-term administration of NSAIDs, an acceptable outcome was achieved in both cases.

The fifth case was presented with a recurrent lameness 12 months after surgery. The elbow joint was limited in the ROM, without palpable pain. However, pain could be elicited by manipulation of the ipsilateral shoulder joint. Both joints were evaluated arthroscopically. In the elbow, a small coronoid tip fragmentation had formed about $2 \mathrm{~mm}$ cranial to the ulnar implant. Caudal to the ulnar implant a groove-like chondral and subchondral bone defect was present, with the eburnated subchondral bone been partially covered with a grayish decoloration. This lesion corresponded to the contact area of the HImpl, which did not appear to be obviously mal-positioned or mal-oriented, both in arthroscopy and bi-planar radiographs (medial opening angle of $17^{\circ}$ and ICPS of $83^{\circ}$ ). The ulnar implant also manifested signs of superficial wear as well as slight plastic deformation. Beside arthroscopic fragment removal, no further treatment was performed. The shoulder joint showed the presence of osteoarthritis, mild tendinopathy of the subscapularis tendon, and elongation of the medial glenohumeral ligament. Following one intra-articular ACP injection into the shoulder joint, lameness improved significantly 6 weeks later. There was no evidence of pain on manipulation of the shoulder joint and the dog achieved an acceptable functional outcome.

Distal radial diaphyseal fracture occurred intraoperatively in one case while exerting excessive rotational moment with the carpus held in $90^{\circ}$ flexed position for improved medial joint space opening. The fracture was reduced and fixed in a minimally invasive plate osteosynthesis fashion with a 3.5-mm locking compression plate. Healing was uneventful and an acceptable outcome was documented in this case at the last follow-up 15 months after surgery, having a LOAD score of 25.

Proximal obliqueulna fracture occurred intraoperatively in one case while exerting forced supination using the bone clamp at the olecranon as lever. The fracture was reduced and fixed with two cortical screws in lag fashion. No further complication occurred and the dog achieved an acceptable outcome at the last followup examination 2 months after surgery with a LOAD score of 11 .
Septic arthritis and implant loosening occurred in one case. This dog initially received two ulnar implants due to extensive ulnar cartilage erosion and was represented 6 weeks after the surgery with severe lameness and pain. No signs of implant loosening or migration were apparent on radiographs. Diagnosis of septic arthritis was based on synovial cytological analysis and later a highly sensitive Staphylococcus intermedius genus confirmed by microbial culture. Second look arthroscopy revealed loosening of the cranial UImpl, which could be easily manipulated with a hook probe. The loose UImpl was removed arthroscopically and the joint was thoroughly lavaged. The second UImpl was left in place as it appeared completely stable and well-integrated. The same applied to the HImpl. Systemic antibiotic treatment with Amoxicillin-Clavulanate (Synulox ${ }^{\circledR}$, Zoetis, Germany) was initiated and continued for 4 weeks. Cytological cell count dropped to values below 1000 cells/ $\mu \mathrm{L}$ with three negative consecutive joint taps. Within 8 weeks after revision, surgery limb function improved significantly. Third-look arthroscopy was performed 3 months after second-look and fibrocartilage infill of the former implant bed was noted. Both remaining implants appeared stable and well-integrated. However, the HImpl had grinded along its axial border into the MCP at a depth of about $0.5 \mathrm{~mm}$ (Fig. 8A). That groove extended from the tip of the MCP over the retained caudal UImpl, $3 \mathrm{~mm}$ caudal to it. The joint was accessed via the caudomedial approach and the implant bed of the previously explanted implant was over reamed with the large sized reamer (previously a medium UImpl) and a large UImpl was implanted (Fig. 8B). Using a high-speed burr, the groove at the MCP was leveled out to achieve a smooth joint surface. No further treatment was performed. At the last follow-up examination 4 months after revision surgery and 10 months after initial surgery, respectively, lameness improved significantly and the dog achieved an acceptable outcome with a LOAD score of 23.

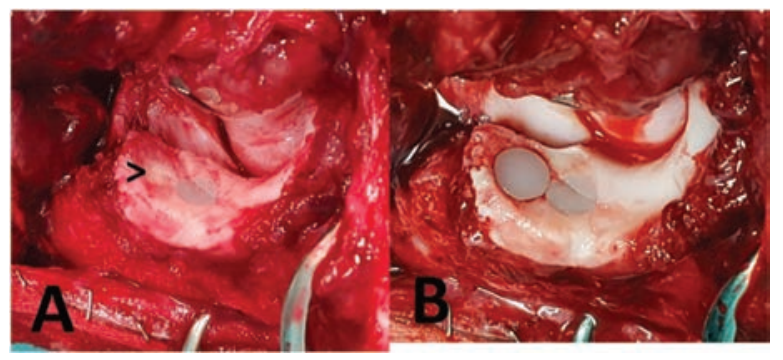

Fig. 8. Revision surgery in a case with septic arthritis and implant loosening 8 weeks after removal of the cranial ulnar implant. (A) Groove-like chondral and subchondral defect with signs of wear of the remaining implant, arrowfibrocartilage infill of the former implant bed and (B) Over reamed bed of the initial implant with an implanted largesized ulnar implant. 


\section{Discussion}

A total of 52 CUEs over a 3-year period between February 2016 and November 2018 were performed in 44 dogs. A successful outcome (acceptable and full function) was achieved in $47 / 48$ (98\%) of the elbows treated, which is comparable to what has been previously reported (88\%-95\%) (Cook et al., 2015; Walmsley and Fitzpatrick, 2018). However, we did not achieve the same proportion of full functional outcomes as previously described, making the CUE procedure in our hands less effective in returning the affected dogs to normal levels of activity. This might be related to the observation that increased age was correlated negatively with clinical outcome in our case series, suggesting that a lower proportion of older dogs achieved full function when compared to younger dogs. This could in part explain the difference to the series reported by Cook et al. (2015), which operated on dogs in average 3 years younger. Another explanation might be the shorter follow-up time in the present study (7.4 months vs. 10 months). Assuming that full rehabilitation following the CUE procedure might take longer in older patients with a postulated higher degree of chronicity of DJD, older patients might not have had enough time to exploit the full potential of the CUE procedure. Finally, older dogs might have more extensive/advanced MCompD which could pose a greater challenge within the context of partial resurfacing inherent to the CUE system. Unfortunately, the degree and extent of cartilage degeneration were not consistently documented in detail, limiting our ability to draw a strong conclusion.

The mean LOAD questionnaire score documented by the owners was on average 20.1 (SD: 7.0) 23.7 (SD: 7.6) months post-operatively, which falls into the category of moderately affected osteoarthritic patients. This is in accordance with the overall successful outcome documented by the surgeons. Unfortunately, due to the retrospective nature of the study preoperative LOAD scores were not available making judgment on the efficacy of the procedure to ameliorate lameness speculative.

The overall complication rate of $21 \%$ in our cohort is lower than in previous reports (38\% and 39\%) where osteotomy of the medial epicondyle was performed (Cook et al., 2015; Walmsley and Fitzpatrick, 2018). When compared to a recent report using a similar caudo-medial approach, the overall complication rates are identical (Walmsley and Fitzpatrick, 2018), which supports our hypothesis that by using a caudomedial approach, complication rates could be lowered. Especially minor complications, which occurred only in $6 \%$ of the cases, are significantly less frequent than what has been previously observed (27\%) (Cook et al., 2015). In consequence, we conclude that the caudomedial approach should be the first choice when performing the CUE procedure, as it reduces approachrelated complications. In respect to the variants of the approach, neither tenotomy of the flexor carpi ulnaris muscle nor osteotomy of the olecranon ridge appears to be significantly better. The addition of a BURP was subjectively felt to improve access to, and visualization of, the medial compartment. The same applies for application of a bone clamp at the proximal ulna serving as a lever arm to facilitate pronation. However, with two intra-operative ulnar fractures, the latter bears a significant risk of intra-operative fracture, which occurred in two of our cases, and, therefore, use of the clamp as a lever has to be done with extreme caution. In the present study, major complications occurred in eight cases (15\%), which is higher than previously reported (4\%-12\%) (Cook et al., 2015). However, because we defined persistent lameness and pain 6 months or later after surgery, necessitating second-look arthroscopy ( $n=5 ; 9.5 \%)$, to be a major complication, the "true" incidence of major complications according to Cook et al. (2010) would have been 6\%.

In two cases, full-thickness cartilage loss was presented caudal to the ulnar implant on the secondlook arthroscopy, indicating erosive wear of the HImpl at the ulnar trochlea. It is interesting to note that both elbows had relatively low preoperative IEWG scores as well as a good ROM. After implantation of one or two additional ulnar implants, respectively, the lameness improved significantly and no signs of pain on palpation were apparent in either case at the followup examination. The obvious improvement in clinical function following implantation of the additional implant(s) leads us to the conclusion, that under some circumstances, resurfacing with only one implant might not be enough. Whether this is related to an increased ROM in dogs with mild degenerative joint disease, allowing the HImpl to contact caudal to the ulnar implant position, or if this is the result of some form of technique related impingement of the HImpl on the ulna is uncertain. However, both on arthroscopy and on radiographic examination, implant position appeared to be correct. In another two cases, progressive bone eburnation with a groove-shaped bone defect along the MCP, as well as signs of polyethylene implant wear and/or plastic deformation, was evident on second-look arthroscopy. Radiographic examination was uneventful in both cases, making more advanced imaging, preferably arthroscopy necessary to pick up this type of complication. We suspect the axial border of the HImpl to have caused those grinding lesions. Because the majority of the humeral implants were not implanted parallel to the ulnar implant, we expected significant obliquity of the HImpl to be the underlying cause. However, when compared to the median deviation of $10.8^{\circ}$, both implants appeared to be properly oriented. Overall, the degree of HImpl mal-angulation did not correlate with the functional outcome or the occurrence of complications in our case series. This should not be taken as a proof that HImpl position and/or orientation are not of clinical significance, because the small 
sample size as well as the way we assessed implant orientation, which might not mimic the true contact situation during weight-bearing (Burton et al., 2013; Goodrich et al., 2014), are considerable bias. Ex vivo biomechanical testing of implant contact following CUE procedure, as well as prospective evaluation of implant contact and related osteochondral or implant wear in a larger number of clinical cases by means of second-look arthroscopy is warranted. For the first time, we report on septic arthritis and related implant loosening, with the latter being undetectable on the follow-up radiographs. After thorough lavage of the joint, and explantation of the loose implant and subsequent antimicrobial therapy, the infection was resolved successfully despite the retained humeral and second ulnar implant. Over reaming and implantation of a larger implant proved to be an effective revision strategy supporting the statement of Franklin et al. (2014) that CUE is a bone sparing procedure offering more exit strategies than just implant removal with a fusion of the joint or limb amputation in case of septic loosening.

\section{Limitations}

With 52 elbow joints investigated, statistical analysis of causative relation might not reach significance because of a type 2 error. Therefore, the absence of significant differences or correlations should be interpreted with caution.

Another limitation of this study is the relatively short follow-up period. In 15 cases, the recommended 6 months follow-up was not achieved and four cases never returned for a follow-up examination. Especially with respect to wear and implant loosening, it is well known that with increased follow-up time the incidence of such findings will increase. Having second-look arthroscopy performed only in cases with treatment failure has limited our observation to the subset of cases with obvious clinical signs. We strongly believe that even in cases with acceptable and even full-functional outcome, wear and bony eburnation is likely to be present, potentially causing clinical symptoms over a longer period of time.

Even though the current study focused on type and frequency of complications when performing the CUE procedure using a caudo-medial approach, reporting functional outcome, especially in relation to the occurrence of complications, is of high clinical value. However, due to the retrospective nature of the study, limited compliance of the owners to provide LOAD scores, as well as the lack of objective gait analysis, we would consider our findings to be of preliminary value.

\section{Conclusion}

The CUE procedure appears to be an effective treatment option for patients with MCompD. Older dogs might be at risk of having an inferior clinical outcome when compared to young patients. The reason for this is unknown and will have to be evaluated in future studies. The slightly modified caudo-medial approach described in this study provides appropriate access to the medial compartment and decreased the overall complication rate and especially minor complications by comparison to previous case series using the medial approach (Cook et al., 2015; Walmsley and Fitzpatrick, 2018). However, emerging evidence of contact lesions of the HImpl along the ulnar contact area and extension of osteochondral defects as well as polyethylene implant wear following surgery warrant close attention in the future.

\section{Conflict of interest}

Four of the authors are involved in CUE training courses and paid consultants of Arthex Vet Systems. Neither of the authors receives any royalties related to sales of CUE implants or instrumentation. No financial support in any form was provided in relation to the study.

\section{Authors contribution}

Bayer K.: writing of the manuscript, data collection, and retrospective data analysis. Böttcher P.: Supervisor, study design. Winkels P., Andreoni A.A., Schmierer P., Pozzi A.: CUE surgeries and collection of the data, corrections of the manuscript. Rohwedder T.: corrections of the manuscript.

\section{References}

Amman, A. and Wendelburg, K. 2010. A novel approach to the caudomedial aspect of the canine elbow via a parasagital osteotomy of the medial ridge of the olecranon. Vet. Surg. Scientific Presentation Abstracts E27, pp: 39.

Burton, N.J., Warren-Smith, C.M.R., Roper, D.P. and Parsons, K.J. 2013. CT Assessment of the influence of dynamic loading on physiological incongruency of the canine elbow. J. Small Anim. Pract. 54(6), 291-298.

Cook, J.L., Schulz, K.S., Karnes, G.J., Franklin, S.P., Canapp, S.O. Jr., Lotsikas, P.J., Fitzpatrick, N., Wheeler, J.L., Stiffler, K.S., Gillick, M., Cross, A.R., Walls, C.M., Albrecht, M.R., Williams, N., Crouch, D.T., Lewis, D.D., Pozzi, A. and Ridge, P. 2015. Clinical outcomes associated with the initial use of the canine unicompartmental elbow (CUE) arthroplasty system (R). Can. Vet. J. 56(9), 971977.

Cook, J.L., Evans, R., Conzemius, M.G., Lascelles, B.D.X., McIlwraith, C.W., Pozzi, A., Clegg, P., Innes, J., Schulz, K., Houlton, J., Fortier, L., Cross, A.R., Hayashi, K., Kapatkin, A., Brown, D.C. and Stewart, A. 2010. Proposed definitions and criteria for reporting time frame, outcome, and complications for clinical orthopedic studies in veterinary medicine. Vet. Surg. 39(8), 905-908.

Coppieters, E., Gielen, I., Verhoeven, G., Van Vynckt, D. and Van Ryssen, B. 2015. Erosion of the medial compartment of the canine elbow: occurrence, diagnosis and currently available treatment options. Vet. Comp. Orthop. Traumatol. 28(1), 9-18. 
Farrell, M., Heller, J., Sollano, M. and Sparrow, T. 2014. Does radiographic arthrosis correlate with cartilage pathology in Labrador retrievers affected by medial coronoid process disease? Vet. Surg. 43, 155-165.

Fitzpatrick, N., Yeadon, R., Smith, T. and Schulz, K. 2009a. Techniques of application and initial clinical experience with sliding humeral osteotomy for treatment of medial compartment disease of the canine elbow. Vet. Surg. 38(2), 261-278.

Fitzpatrick, N., Smith, T.J., Evans, R.B. and Yeadon, R. 2009b. Radiographic and arthroscopic findings in the elbow joints of 263 dogs with medial coronoid disease. Vet. Surg. 38(2), 213-223.

Franklin, S.P., Schulz, K.S., Karnes, J. and Cook, J.L. 2014. Theory and development of a unicompartmental resurfacing system for treatment of medial compartment disease of the canine elbow. Vet. Surg. 43(7), 765-773.

Goodrich, Z.J., Norby, B., Eichelberger, B.M., Friedeck, W.O., Callis, H.N., Hulse, D.A., Kerwin, S.C., Fox, D.B. and Saunders, W.B. 2014. Thoracic limb alignment in healthy labrador retrievers: evaluation of standing versus recumbent frontal plane radiography. Vet. Surg. 43(7), 791-803.

Hanson, S.P., Peck, J.N., Berry, C.R., Graham, J. and Stevens, G. 2006. Radiographic evaluation of the Zurich cementless total hip acetabular component. Vet. Surg. 35(6), 550-558.
Ohlerth, S., Tellhelm, B. and Amort, K. 2016. Explanation of the IEWG Grading System. 30th Annual Meeting IEWG 2016 Vienna, Austria.

Smith, Z.F., Wendelburg, K.L., Tepic, S., Stover, S.M., Garcia-Nolen, T., Stearns, P.B. and Hayashi, K. 2013. In vitro biomechanical comparison of load to failure testing of a canine unconstrained medial compartment elbow arthroplasty system and normal canine thoracic limbs. Vet. Comp. Orthop. Traumatol. 26(5), 356-365.

Van Ryssen, B. and Van Bree, H. 1997. Arthroscopic findings in 100 dogs with elbow lameness. Vet. Rec. 140(14), 360-362.

Vermote, K.A.G, Bergenhuyzen, A.L.R, Gielen, I., Van Bree, H., Duchateau, L. and Van Ryssen, B. 2009. Elbow lameness in dogs of six years and older. Vet. Comp. Orthop. Traumatol. 23(1), 43-50.

Walmsley, D. and Fitzpatrick, N. 2018. Complications associated with Canine Unicompartmental Elbow (CUE) arthroplasty in 91 elbows via medial epicondylar osteotomy compared a caudomedial approach. 5th World Veterinary Orthopaedic Congress ESVOT Proceedings 2018, pp: 507-508.

Walton, B., Innes, J., Lascelles, B.D.X. and Belshaw, Z. 2017. Validated outcomes measures for canine osteoarthritis. Vet. Surg. 46(3), 341-342.

Winkels, P. and Busch, C. 2018. Caudo-medial approach in CUE - intra and postoperative complications. 5th World Veterinary Orthopaedic Congress ESVOT Proceedings 2018, pp: 519-520. 